\title{
GENERALIZED PN MANIFOLDS AND SEPARATION OF VARIABLES
}

\author{
FERNAND PELLETIER and PATRICK CABAU \\ LAMA, Université de Savoie \\ F-73376 Le Bourget-du-Lac Cedex, France \\ E-mail:pelletier@univ-savoie.fr,patrickcabau@yahoo.fr
}

\begin{abstract}
The notion of generalized PN manifold is a framework which allows one to get properties of first integrals of the associated bihamiltonian system: conditions of existence of a bi-abelian subalgebra obtained from the momentum map and characterization of such an algebra linked with the problem of separation of variables.
\end{abstract}

1. Introduction. A geometric characterization of integrable Hamiltonian systems was given by Magri and Morosi in the famous paper [15] written in 1984.

A Hamiltonian system on an even dimensional $(2 m)$ manifold $M$ corresponds to a Poisson tensor of maximal rank associated with a smooth function $H$ (called Hamiltonian) whose gradient does not identically vanish. Such a system is integrable if one can find another Poisson structure $Q$ compatible with $P$ (i.e. such that $P+Q$ is still a Poisson tensor) for which the associated recursion operator $N=Q P^{-1}$ has $m$ pairwise distinct real eigenvalues $\lambda_{1}, \ldots, \lambda_{m}$ of order 2 , first integrals in involution $\left(P\left(d \lambda_{i}, d \lambda_{j}\right)=0\right)$ and functionally independent on a dense open set. Moreover, one can find functions $\mu_{1}, \ldots, \mu_{m}$ by quadrature such that the inverse $\omega$ of $P$ (which is a symplectic form) may be written as $\omega=\sum_{i=1}^{m} d \lambda_{i} \wedge d \mu_{i}$ and where the matrix of $N^{*}$ has a diagonal form.

It is well known (cf. [15], [5] for example) that the $m$ maps $I_{k}=\frac{1}{k} \operatorname{trace} N^{k}(k=$ $1, \ldots, m)$ are in involution with respect to both structures $P$ and $Q$ and give rise to a Lenard recursion chain: $N^{*}\left(d I_{k}\right)=d I_{k+1}$.

This bihamiltonian scheme may be generalized to:

- odd dimensional $(2 m+1)$ manifolds which are an adapted framework for a lot of inte-

2000 Mathematics Subject Classification: 37J05, 37J15, 37J20, 53B50, 53D05, 53D17, 53Z05, $53 \mathrm{Z} 20$.

Key words and phrases: bihamiltonian structures, integrable systems, PN manifold, recursion operator, separation of variables, bi-abelian algebra, momentum map, singularities, stratifications.

The paper is in final form and no version of it will be published elsewhere. 
grable Hamiltonian systems which can be written as Gel'fand-Zakharevich systems (cf. [8]): in this case, the $2 m$-rank Poisson tensors $P$ and $Q$ give rise to a Poisson pencil $P_{\lambda}=Q-\lambda P, \varphi \in \mathbb{R} \cup\{\infty\}$; the generic symplectic leaf of the pencil has dimension $2 m$ and the intersection of all the symplectic leaves are generically Lagrangian submanifolds. Moreover, there exist Casimir functions $c_{\lambda}$ for the Poisson pencil, i.e. such that $d c_{\lambda} \in \operatorname{ker} P_{\lambda}$, which give rise to first integrals ([14]).

- infinite dimensional manifolds: the famous nonlinear PDE Korteweg de Vries can be written as a bihamiltonian system ([13], [6]). Using the associated recursion operator, one can produce an infinite sequence of first integrals ([20]). Other PDEs, such as Camassa-Holm and Hunter-Saxon equations, may be seen as bihamiltonian systems on the dual of the Virasoro algebra ([10]) (the first Poisson tensor corresponds to the Lie Poisson structure).

In this paper, we consider the framework of generalized PN manifolds, i.e. finite dimensional manifolds endowed with both a Poisson structure $P$ and a Nijenhuis tensor $N([N, N]=0)$ called recursion operator, defined on the involutive distribution $\operatorname{im} P$ and compatible with $P$ (i.e. the tensor $P^{\prime}=N P$ is still a Poisson tensor). This notion illustrated with various examples, is introduced in section 2 . When $P$ is of maximal rank, the manifold may be endowed with a $\omega N$ structure ([5]).

If one considers a generalized PN manifold $(M, P, N)$, it is obvious that $M$ is not necessarily even dimensional; moreover, in general, the Poisson tensor $P$ has singularities of rank. $N$ has also singularities and its eigenvalues can be real or complex; furthermore the number of such distinct eigenvalues depends on the point of the manifold. We can completely describe the singularities of $(P, N)$ in terms of rank of $P$ and Jordan normal form of $N$ described by a numerical invariant called symbol. With this invariant we can define a stratification in the set of pairs $(P, N)$ (section 3 ). Transversality to this stratification gives rise to the notion of genericity ( $\S 4.2$ ). Moreover, in the analytic context, each set where the symbol of $(P, N)$ at the point of the manifold is constant may be stratified. The set on which the symbol is "maximal" is an open set. On such a set, Turiel has built an adapted local coordinates system where $P$ has a symplectic expression and $N$ a diagonal form.

In another way, to a Poisson structure $P$, we can associate a bracket $\{., .\}_{P}$ on the set $\mathcal{E}$ of the $C^{\infty}$ functions on $M:\{f, g\}_{P}=\langle d f, P d g\rangle$ (where $P: T^{*} M \rightarrow T M$ ). So, $\mathcal{E}$ may be endowed with a Lie algebra structure. On a generalized PN manifold we get a pair of such Lie algebra structures $\left(\mathcal{E},\{., .\}_{P}\right)$ and $\left(\mathcal{E},\{., .\}_{N P}\right)$. If we come back to the context of the first integrals $f_{1}, \ldots, f_{n}$, these functions generate two structures of abelian sub-algebra called bi-abelian sub-algebra; a hamiltonian which belongs to this algebra is completely integrable.

On a $2 m$-dimensional manifold, if we consider the generic context or the analytic one with the existence of an open set where $N$ has exactly $m$ distinct real or complex conjugate eigenvalues (of multiplicity 2) (which is always true in the generic case), we have information about the existence of the bi-abelian sub-algebra (theorem 5.1) and we get a characterization (theorem 6.1) of such an algebra generalizing the results given by Falqui and Pedroni in [5]: 
1. The functions $\left\{I_{k}=\frac{1}{k} \operatorname{trace} N^{k}\right\}_{k=1, \ldots, m}$ are independent on an open set whose complement is a stratified set of codimension 2. Moreover, these functions generate a bi-abelian sub-algebra.

2. Let $f_{1}, \ldots, f_{m}$ be independent functions on a dense open set. The following propositions are equivalent:

(a) $f_{1}, \ldots, f_{m}$ generate a bi-abelian sub-algebra;

(b) on a dense open set, $d f_{1}, \ldots, d f_{m}$ generate a bi-lagrangian foliation;

(c) on each open set where the symbol is constant $f_{1}, \ldots, f_{m}$ are "separable" in the Turiel adapted coordinates $\left(x_{i}, y_{i}\right)$ of $(P, N)$; the separability is associated with the existence of functions $\Phi_{j}, j=1, \ldots, m$ such that

$$
\Phi_{j}\left(x_{i}, y_{i}, f_{1}, \ldots, f_{m}\right)=0, \quad j=1, \ldots, m, \quad \operatorname{det}\left[\frac{\partial \Phi_{j}}{\partial f_{i}}\right] \neq 0,
$$

and we can apply the classic Hamilton-Jacobi method of separation of variables.

2. Generalized PN manifolds. Let $M$ be a manifold of finite dimension $n$.

\subsection{Tensors and brackets}

2.1.1. Poisson tensor and bracket. A twice contravariant and skew-symmetric tensor field $P$ is a Poisson tensor iff its Schouten bracket $[P, P]$ vanishes where $[P, P] \in \underline{\Lambda^{3} T M}$ is defined by:

$$
\langle\alpha,[P, P](\beta, \gamma)\rangle=\underset{\alpha, \beta, \gamma}{\sigma}\left\langle L_{P \alpha} \gamma, P \beta\right\rangle
$$

where $\underset{\alpha, \beta, \gamma}{\sigma}$ stands for the sum of the cyclic permutations of $\alpha, \beta$ and $\gamma$.

The components of the Poisson tensor satisfy the cyclic condition:

$$
\sum_{l}\left(P^{i l} \frac{\partial P^{j k}}{\partial x^{l}}+P^{j l} \frac{\partial P^{k i}}{\partial x^{l}}+P^{k l} \frac{\partial P^{i j}}{\partial x^{l}}\right)=0
$$

The associated distribution $\operatorname{im} P$ is involutive because we have for closed forms $\alpha$ and $\beta$ :

$$
[P \alpha, P \beta]=P\left(L_{P \beta} \alpha-L_{P \alpha} \beta+d\langle\beta, P \alpha\rangle\right)
$$

but, generically, not of constant rank. Nonetheless, at each point, there exists an integral submanifold of maximal dimension which is tangent to the distribution.

On another way, this Poisson tensor field gives rise to a Lie algebra structure on the set of the smooth functions on $M$ for the bracket $\{., .\}_{P}$ defined by:

$$
\{f, g\}_{P}=\langle d f, P(d g)\rangle
$$

where $P$ is seen as a morphism $T^{*} M \rightarrow T M$.

If the Poisson tensor is of maximal rank on an even dimensional manifold $(n=2 m)$, this manifold may be endowed with a symplectic structure given by $P^{-1}$ (the symplectic form is seen as a morphism $\left.T M \rightarrow T^{*} M\right)$. 
2.1.2. Nijenhuis tensor. The torsion $T(N)$ of a $(1,1)$-tensor field $N$ is defined by:

$$
T(N)(X, Y)=[N X, N Y]-N[N X, Y]-N[X, N Y]+N^{2}[X, Y] .
$$

Such a tensor is called a Nijenhuis tensor iff $T(N)=0$ and, in this case, the associated distribution $\operatorname{im} N$ is integrable.

2.1.3. Compatible tensors. For a Poisson tensor $P$ and a Nijenhuis tensor $N$ we define the bracket $[P, N]$ :

$$
[P, N](\alpha, X)=\left(L_{P \alpha} N\right) X-P . L_{X}\left(N^{*} \alpha\right)+P L_{N X} \alpha .
$$

If $N P$ is skew-symmetric and if $[P, N]=0$, the tensor $N P$ is a Poisson tensor (cf. [15]).

2.2. Generalized $P N$ manifold. We now define the main structure we are going to use here.

Definition 2.1. A generalized $P N$ manifold is a manifold $M$ endowed with a Poisson tensor $P$, a Nijenhuis tensor $N$ defined on $\operatorname{im} P$, such that $N P$ is skew-symmetric and $[P, N]$ vanishes.

Remark 2.2. When the Poisson tensor $P$ is of maximal rank, $P^{-1}$ corresponds to a symplectic 2-form (as seen above) and so $M$ may be endowed with an $\omega N$ structure ([15], [5]), which is a particular case of a $P N$ manifold ([11], [16]).

REMARK 2.3. If $(M, P, N)$ is a generalized $\mathrm{PN}$ manifold, the tensor $N P$ is a Poisson tensor too and $[N P, N]$ vanishes. So we get, by induction, a hierarchy of such structures defined by $P_{j}=N^{j} P$.

\subsection{Examples of generalized $P N$ manifolds.}

2.3.1. Cotangent bundle. As is well known, a Poisson structure $P$ of maximal rank is well defined on the cotangent bundle of a manifold $S$ where a symplectic form $d \alpha$ naturally exists ( $\alpha$ is the canonical Liouville form). If we consider a Nijenhuis tensor $L$ on $S$, one can define, as shown in [9], a second Poisson structure $Q$ on $T^{*} S$, compatible with $P$, whose bracket $\{., .\}_{Q}$ has, in the fibred coordinates, the following expression:

$$
\left\{q_{i}, q_{j}\right\}_{Q}=0, \quad\left\{q_{i}, p_{j}\right\}_{Q}=-L_{j}^{i}, \quad\left\{p_{i}, p_{j}\right\}_{Q}=\left(\frac{\partial L_{j}^{k}}{\partial q_{i}}-\frac{\partial L_{i}^{k}}{\partial q_{j}}\right) p_{k}
$$

$N$, complete lifting of $L$ as defined in [30], is the recursion operator for this bihamiltonian structure.

$\left(T^{*} S, P, N\right)$ is a generalized $\mathrm{PN}$ manifold and may be endowed with an $\omega N$ structure.

2.3.2. A case of integrability of the Hénon-Heiles system. Let us consider the HénonHeiles dynamical system (which is a generalization of the oscillator) on $M=\mathbb{R}^{4}$ defined by the Hamiltonian

$$
H=\frac{1}{2} A q_{1}^{2}+\frac{1}{2} B q_{2}^{2}+\frac{1}{2} p_{1}^{2}+\frac{1}{2} p_{2}^{2}+\lambda q_{1}^{2} q_{2}+\mu q_{2}^{3} .
$$

The case $\mu=2 \lambda$ is one of the 3 cases of integrability (for $\lambda=0$, we obtain the oscillator: $\left.H=\frac{1}{2} A q_{1}^{2}+\frac{1}{2} B q_{2}^{2}+\frac{1}{2} p_{1}^{2}+\frac{1}{2} p_{2}^{2}\right)$. 
The second first integral is given by:

$$
F=\frac{1}{8}\left[\left(4 A-B-4 \lambda q_{2}\right) p_{1}^{2}+4 \lambda q_{1} p_{1} p_{2}+q_{1}^{2}\left(4 A^{2}-A B+4 A \lambda q_{2}+\lambda^{2} q_{1}^{2}+4 \lambda^{2} q_{2}^{2}\right)\right] .
$$

So, for $\lambda \neq 0$, the system can be written in a bihamiltonian form ([3]) with the following compatible Poisson tensors:

$$
P:=\left(\begin{array}{cccc}
0 & 0 & -1 & 0 \\
0 & 0 & 0 & -1 \\
1 & 0 & 0 & 0 \\
0 & 1 & 0 & 0
\end{array}\right) \text { and } Q:=\left(\begin{array}{cccc}
0 & 0 & 0 & -\frac{x_{1}}{2 \lambda} \\
0 & 0 & -\frac{x_{1}}{2 \lambda} & -\frac{x_{2}}{\lambda}+\frac{C}{4 \lambda^{2}} \\
0 & \frac{x_{1}}{2 \lambda} & 0 & \frac{x_{3}}{2 \lambda} \\
\frac{x_{1}}{2 \lambda} & \frac{x_{2}}{\lambda}-\frac{C}{4 \lambda^{2}} & -\frac{x_{3}}{2 \lambda} & 0
\end{array}\right)
$$

where $C=4 A-B, x_{1}=q_{1}, x_{2}=q_{2}, x_{3}=p_{1}, x_{4}=p_{2}$.

The matrix of the recursion operator $N:=Q P^{-1}$ is

$$
\left(\begin{array}{cccc}
0 & \frac{1}{2 \lambda} x_{1} & 0 & 0 \\
\frac{1}{2 \lambda} x_{1} & -\frac{1}{4} \frac{C}{\lambda^{2}}+\frac{1}{\lambda} x_{2} & 0 & 0 \\
0 & -\frac{1}{2 \lambda} x_{3} & 0 & \frac{1}{2 \lambda} x_{1} \\
\frac{1}{2 \lambda} x_{3} & 0 & \frac{1}{2 \lambda} x_{1} & -\frac{1}{4} \frac{C}{\lambda^{2}}+\frac{1}{\lambda} x_{2}
\end{array}\right)
$$

$\left(\mathbb{R}^{4}, P, N\right)$ is a generalized $\mathrm{PN}$ manifold where $P$ is of maximal rank and so may be endowed with an $\omega N$ structure.

2.3.3. Structures linked with 'homogeneous' Poisson structures. The example we describe here is constructed with a pair of Poisson structures $P_{0}$ and $P_{r}, r \in \mathbb{N}^{*}$, on $\mathbb{R}^{2 m}$ where $P_{0}$ and $P_{r}$ are two homogeneous structures linked with the vector field $X$ $\left(L_{X} P_{0}=\alpha P_{0}\right.$ and $L_{X} P_{r}=\beta_{r} P_{r}, \alpha$ and $\beta_{r}$ are constants) (cf. [26]).

Let $x_{1}, \ldots, x_{2 m}$ be the coordinates on $\mathbb{R}^{2 m}$, we consider $P_{0}=\left(\begin{array}{cc}0_{m} & -\operatorname{Id}_{m} \\ \operatorname{Id}_{m} & 0_{m}\end{array}\right)$ and $P_{r}=\left(\begin{array}{cc}0_{m} & -\Lambda_{m, r} \\ \Lambda_{m, r} & 0_{m}\end{array}\right)$ where $\Lambda_{m, r}$ is the diagonal matrix $m \times m$ constructed with $x_{1}^{r}, \ldots, x_{m}^{r}$. The recursion operator is diagonal and its double eigenvalues are $x_{1}^{r}, \ldots, x_{m}^{r}$.

2.3.4. Lie groups. Structures of generalized PN manifolds on Lie groups can be obtained by using the results given in [15]. On a Lie group $G$, we consider two compatible tensors $P$ and $Q$ where the first one is invertible and left-invariant (such as $P^{-1}$ ) and where $Q$ is right-invariant. In this case, the recursion operator is $N=Q P^{-1}$.

So the research of a pair $(P, N)$ where $P$ is an invertible Poisson tensor and $N$ is Nijenhuis is linked with the research of $P_{e}$ and $\Omega_{e}$ (where $e$ stands for the identity of $G$ ) only fulfilling algebraic conditions (skew-symmetry and Jacobi identity).

2.3.5. Dual of a Lie algebra. Let $G$ be a Lie group where $e$ stands for the identity, $\mathcal{G}$ its Lie algebra and $\mathcal{G}^{*}$ the dual of this algebra. We shall use the natural identifications $\mathcal{G} \simeq T_{\alpha}^{*} \mathcal{G}^{*}$ and $\mathcal{G}^{*} \simeq T_{\alpha} \mathcal{G}^{*}$. 
$\mathcal{G}^{*}$ is endowed with the usual Lie-Kirillov-Poisson structure $K$ defined by:

$$
K_{\alpha} X=\operatorname{ad}_{X}^{*} \alpha .
$$

Modulo these identifications and thanks to the cocycle $P_{e}: \mathcal{G}^{*} \rightarrow \mathcal{G}$, we can construct a presymplectic constant tensor on $\mathcal{G}^{*}$

$$
\Omega_{\alpha} \beta=P_{e} \beta
$$

Let $H$ be the connected Lie subgroup whose Lie algebra is

$$
\mathcal{H}=P_{e}\left(\mathcal{G}^{*}\right) .
$$

We can get a Poisson structure $\Pi$ of maximal rank and a compatible presymplectic structure $\omega$ on $\mathcal{H}^{*}=\mathcal{G}^{*} /$ ker $P_{e}$ using a restriction technique (cf. [15]). So $\left(\mathcal{H}^{*}, \Pi, \omega \Pi^{-1}\right)$ is a generalized PN manifold.

2.3.6. Use of a Casimir function; example of the rigid body. If $c$ is a Casimir function for a Poisson structure $P$, i.e. $d c \in \operatorname{ker} P$, and if $\{.,$.$\} is the associated Poisson bracket,$ then the bracket $\{., .\}_{c}=c\{.,$.$\} defines a new Poisson structure and it is possible to$ define a recursion operator on $\operatorname{im} P$ which is a homothety. We then have a generalized PN manifold and the Poisson tensor is not of maximal rank.

As an example, we consider the case of the rigid body. Euler's equations for a body with a fixed point where the 3 principal moments of inertia are the strictly positive and distinct reals $I_{1}, I_{2}$ and $I_{3}$ :

$$
\left\{\begin{array}{l}
\dot{x}_{1}=\left(\frac{1}{I_{2}}-\frac{1}{I_{3}}\right) x_{2} x_{3} \\
\dot{x}_{2}=\left(\frac{1}{I_{3}}-\frac{1}{I_{1}}\right) x_{3} x_{1} \\
\dot{x}_{1}=\left(\frac{1}{I_{1}}-\frac{1}{I_{2}}\right) x_{1} x_{2}
\end{array}\right.
$$

where $x_{1}, x_{2}$ and $x_{3}$ are the components of the angular momentum of the body at its fixed point in the orthogonal frame made by the eigenvectors of the inertia operator (see e.g. [19]).

This system is hamiltonian and the Poisson tensor, corresponding to the linear LiePoisson structure on $\mathfrak{s o}^{*}(3, \mathbb{R})$ dual of the Lie algebra $\mathfrak{s o}(3, \mathbb{R})$ (isomorphic to $\mathbb{R}^{3}$ endowed with the cross-product), is

$$
P=\left(\begin{array}{ccc}
0 & -x_{3} & x_{2} \\
x_{3} & 0 & -x_{1} \\
-x_{2} & x_{1} & 0
\end{array}\right)
$$

It is easy to see that $c:\left(x_{1}, x_{2}, x_{3}\right) \mapsto x_{1}^{2}+x_{2}^{2}+x_{3}^{2}$ is a Casimir function for $P$.

$Q=c P$ is a Poisson structure compatible with $P$ and the matrix of the recursion operator is, in a basis of im $P$, equal to $\left(\begin{array}{cc}x_{1}^{2}+x_{2}^{2}+x_{3}^{2} & 0 \\ 0 & x_{1}^{2}+x_{2}^{2}+x_{3}^{2}\end{array}\right)$. 
One can find another Poisson structure $T$ compatible with $P$ where

$$
T=\left(\begin{array}{ccc}
0 & -\frac{x_{3}}{I_{3}} & \frac{x_{2}}{I_{2}} \\
\frac{x_{3}}{I_{3}} & 0 & -\frac{x_{1}}{I_{1}} \\
\frac{-x_{2}}{I_{2}} & \frac{x_{1}}{I_{1}} & 0
\end{array}\right) .
$$

In this case, it is not possible to find a recursion operator because $\operatorname{ker} P \neq \operatorname{ker} T$.

2.3.7. The Toda lattice. The Toda lattice on $M=\mathbb{R}_{+}^{* 2} \times \mathbb{R}^{3}$, obtained from a Hamiltonian system on $\mathbb{R}^{6}$ via a Flaschka-Manakov transformation ([7], [4]) with coordinates $\left(a_{1}, a_{2}, b_{1}, b_{2}, b_{3}\right)$, may be written as

$$
\left\{\begin{array}{l}
\dot{a}_{1}=a_{1}\left(b_{2}-b_{1}\right), \\
\dot{a}_{2}=a_{3}\left(b_{3}-b_{2}\right), \\
\dot{b}_{1}=2 a_{1}^{2} \\
\dot{b}_{2}=2\left(a_{2}^{2}-a_{1}^{2}\right) \\
\dot{b}_{3}=-2 a_{2}^{2}
\end{array}\right.
$$

This is the following Hamiltonian system

$$
\dot{x}=\widehat{P} d \widehat{H}
$$

where the Poisson tensor $\widehat{P}$, which is of rank $4\left(\operatorname{ker} \widehat{P}=\left\langle d b_{1}+d b_{2}+d b_{3}\right\rangle\right)$ on the 5 dimensional manifold, is given by its matrix:

$$
\frac{1}{4}\left(\begin{array}{ccccc}
0 & 0 & -a_{1} & a_{1} & 0 \\
0 & 0 & 0 & -a_{2} & a_{2} \\
a_{1} & 0 & 0 & 0 & 0 \\
-a_{1} & a_{2} & 0 & 0 & 0 \\
0 & -a_{2} & 0 & 0 & 0
\end{array}\right)
$$

and where the Hamiltonian $\widehat{H}$ is:

$$
\widehat{H}=4\left(a_{1}^{2}+a_{2}^{2}\right)+2\left(b_{1}^{2}+b_{2}^{2}+b_{3}^{2}\right) .
$$

This function is proportional to the trace of the endomorphism $L^{2}$ where $L$ is given by its matrix $\left(\begin{array}{ccc}b_{1} & a_{1} & 0 \\ a_{1} & b_{2} & a_{2} \\ 0 & a_{2} & b_{3}\end{array}\right)$. This Hamiltonian system can be written as a Lax pair

$$
\dot{L}=[L, B]
$$

where $B=\left(\begin{array}{ccc}0 & -a_{1} & 0 \\ a_{1} & 0 & -a_{2} \\ 0 & a_{2} & 0\end{array}\right)$. The flow of this Hamiltonian vector field is isospectral,

i.e. that the eigenvalues of $L$ are independent of $t$.

Remark that

$$
c\left(a_{1}, a_{2}, b_{1}, b_{2}, b_{3}\right)=\operatorname{tr} L=b_{1}+b_{2}+b_{3}
$$

is a Casimir function for the Poisson tensor $\widehat{P}$. 
There exists on $M$ another Poisson tensor $\widehat{Q}$ (see e.g. [18]) whose matrix is:

$$
\frac{1}{4}\left(\begin{array}{ccccc}
0 & -a_{1} a_{2} & a_{1} b_{1} & -a_{1} b_{2} & 0 \\
a_{1} a_{2} & 0 & 0 & a_{2} b_{2} & -a_{2} b_{3} \\
-a_{1} b_{1} & 0 & 0 & a_{1} & 0 \\
a_{1} b_{2} & -a_{2} b_{2} & -a_{1} & 0 & a_{2} \\
0 & a_{2} b_{3} & 0 & -a_{2} & 0
\end{array}\right) .
$$

Because ker $\widehat{Q} \nsupseteq$ ker $\widehat{P}$, a recursion operator $\widehat{N}$ does not exist in this case.

Falqui and Pedroni give in [5] conditions to induce an $\omega N$ structure on a symplectic leaf of $\widehat{P}$. These conditions are linked with the existence of a vector field $Z$ which is transversal to the symplectic foliation of this Poisson tensor and fulfilling the conditions:

$$
Z(c)=1, \quad L_{Z} \widehat{P}=0, \quad L_{Z} \widehat{Q}=Y \wedge Z
$$

for a suitable vector field $Y$.

In our example, $Z=\frac{\partial}{\partial b_{3}}$ and $Y=\frac{a_{2}}{4} \frac{\partial}{\partial a_{2}}$.

\section{Stratification in the space of adapted pairs (bivectors field, endomorphisms field) and symbol}

3.1. Space of pairs (bivector, endomorphism). Let $E$ be a vector space of dimension $n$.

3.1.1. Space $\mathcal{A}$ of adapted pairs (bivector,endomorphism). A bivector of $E$ is a skewsymmetric bilinear form on the dual $E^{*}$ of $E$.

If $w$ is a bivector of $E$, we denote by $w^{\#}: E^{*} \rightarrow E$ the linear map defined by:

$$
\forall \alpha, \beta \in E^{*},\left\langle\beta, w^{\#}(\alpha)\right\rangle=w(\alpha, \beta) .
$$

We shall denote $w$ instead of $w^{\#}$.

The support of the bivector $w, \operatorname{supp} w$, corresponds to the space im $w^{\#}$.

A pair $(w, J)$ where $w$ is a bivector and $J$ an endomorphism of supp $w$ is called adapted if $J \circ w^{\#}$ is still skew-symmetric.

The space $\mathcal{A}_{2 k}$ of the pairs $(w, J)$ where $w$ is of rank $2 k$ is a bundle over $\mathcal{G}_{2 k}(E)$ where $\mathcal{G}_{2 k}(E)$ is the Grassmannian of the $2 k$-linear spaces of $E$.

The fiber over the $2 k$-space $F$ corresponds to the set of the adapted pairs $(w, J)$ such that $\operatorname{supp} w=F$ and $J=w^{-1} w_{1}$ where $w_{1} \in \Lambda^{2} F^{*}$.

REMARK 3.1. If $\mathcal{W}$ is the space of the pairs $\left(w_{1}, w_{2}\right)$ of bivectors of $E$ such that supp $w_{1} \supset$ $\operatorname{supp} w_{2}$, one can associate to any $(w, J) \in \mathcal{A}=\bigcup_{k=0}^{[n / 2]} \mathcal{A}_{2 k}$ an element $(w, J w) \in \mathcal{W}$ and this map is one to one.

3.1.2. Stratification by rank. We define the natural action of the Lie group $G l(E)$ on the set of the bivectors of $E$ by: $(A, w) \mapsto A \bullet w$ where, for any linear forms $\alpha$ and $\beta$ : $A \bullet w(\alpha, \beta)=w\left({ }^{t} A \alpha,{ }^{t} A \beta\right)$; this allows us to define an action of $G l(E)$ on $\mathcal{A}$. In fact, each manifold $\mathcal{A}_{2 k}$ is invariant under this action.

We then construct, on $\mathcal{A}_{2 k}$, a $G l(E)$-invariant stratification $\left\{\Sigma_{c}\right\}$ by the rank of $w_{1}=J w$.

If $(w, J)$ is an adapted pair where $\operatorname{dim} \operatorname{supp} J w=r$, we denote the corank of $J w$ by $c=n-r$. 
Adapting the results obtained in [17] and [24], we get a finite stratification $\Sigma=$ $\left\{\Sigma_{c}\right\}_{0 \leq c \leq n}$ of the space $\mathcal{A}$ where

$$
\Sigma_{c}=\{(w, J) \in \mathcal{A}, \operatorname{corank} J w=c\}
$$

is a regular submanifold $\mathcal{A}_{2 k}$ of codimension $c(c-1) / 2$ and where $\overline{\Sigma_{c}}$ is an algebraic submanifold of $\mathcal{A}$ such that $\overline{\Sigma_{c}}=\bigcup_{c^{\prime} \geq c} \Sigma_{c^{\prime}}$.

3.1.3. Expression in an adapted basis. Here we transpose the results obtained by Turiel ([28]) about the classification of the pairs of 2 -forms.

Proposition 3.2. Let $(w, J)$ be an adapted pair where the minimum polynomial $\mu_{J}$ may be written as $(X-\lambda)^{r}$ where $\lambda$ is a real number. There exists a decomposition of $\operatorname{supp} w$ in a direct sum $\bigoplus_{j=1}^{l} F_{j}$ of linear subspaces, invariant by $J$ of dimension $2 r_{j}$, $r=r_{1} \geq \cdots \geq r_{l}$ and a basis $\left\{e_{j}^{q_{j}}\right\}, q_{j}=1, \ldots, 2 r_{j}, j=1, \ldots, l$ of supp $w_{1}$ where $\left\{e_{j}^{q_{j}}\right\}, q_{j}=1, \ldots, 2 r_{j}$ is a basis of $F_{j}$ such that:

$$
\begin{gathered}
w=\sum_{j=1}^{l}\left(\sum_{k=1}^{r_{j}} e_{j}^{2 k-1} \wedge e_{j}^{2 k}\right), \\
J e_{2 k-2}=\lambda e_{2 k-2}+e_{2 k}, \text { for } k=2, \ldots, r_{j}, \quad J e_{2 r_{j}}=\lambda e_{2 r_{j}}, \\
J e_{2 k+1}=e_{2 k-1}+\lambda e_{2 k+1}, \text { for } k=1, \ldots, r_{j}-1, \quad J e_{1}=\lambda e_{1} .
\end{gathered}
$$

Definition 3.3. The associated basis $(w, J)$ constructed in the above proposition is called the adapted basis to $(w, J)$.

REMARK 3.4. $w_{1}=J w$ is skew-symmetric and we find the expression given in [22]

$$
w_{1}=\sum_{j=1}^{l}\left(\sum_{k=1}^{r_{j}-1} e_{j}^{2 k-1} \wedge e_{j}^{2 k+2}\right)+\lambda w .
$$

Proposition 3.5. Let $(w, J)$ be an adapted pair for which the minimum polynomial $\mu_{J}$ is $(X-\mu)^{r}(X-\bar{\mu})^{r}$ where $\mu=\alpha+i \beta$ is a non real complex number. So there exists a decomposition of $\operatorname{supp} w$ in a direct sum $\bigoplus_{j=1}^{l} F_{j}$ of linear subspaces, invariant by $J$ of dimension $4 r_{j}, r_{j}=r_{1} \geq \cdots \geq r_{l}$ and a basis $\left\{e_{j}^{q_{j}}\right\}, q_{j}=1, \ldots, 4 r_{j}, j=1, \ldots, l$ of $\operatorname{supp} w_{1}$ where $\left\{e_{j}^{q}\right\}, q_{j}=1, \ldots, 4 r_{j}$ is a basis of $F_{j}$ such that:

$$
\begin{gathered}
w=\sum_{j=1}^{l}\left(\sum_{k=1}^{2 r_{j}} e_{j}^{2 k-1} \wedge e_{j}^{2 k}\right), \\
J e_{4 k+1}= \begin{cases}\alpha e_{4 k+1}-\beta e_{4 k+3} & \text { for } k=0, \\
\alpha e_{4 k+1}+\beta\left(e_{4 k+3}-e_{4 k+1}\right) & \text { for } k=1, \ldots, r_{j}-1,\end{cases} \\
J e_{4 k+2}=\alpha e_{4 k+2}+\beta e_{4 k+4} \text { for } k=0, \ldots, r_{j}-1, \\
J e_{4 k+3}=\alpha e_{4 k+3}+\beta e_{4 k+1} \text { for } k=0, \ldots, r_{j}-1, \\
J e_{4 k+4}= \begin{cases}\alpha e_{4 k+4}-\beta\left(e_{4 k+2}-e_{4 k+6}\right) & \text { for } k=0, \\
\alpha e_{4 k+4}-\beta e_{4 k+2} & \text { for } k=1, \ldots, r_{j}-1 .\end{cases}
\end{gathered}
$$

REMARK 3.6. Once again we find the expression of the bivector $w_{1}=J w$ given in [22]

$$
w_{1}=\alpha \cdot w+\beta\left[\sum_{j=1}^{l}\left(\sum_{k=0}^{r_{j}-1} e_{j}^{4 k+1} \wedge e_{j}^{4 k+4}+e_{j}^{4 k+2} \wedge e_{j}^{4 k+3}+\sum_{k=0}^{r_{j}-2} e_{j}^{4 k+3} \wedge e_{j}^{4 k+6}\right)\right]
$$


3.1.4. Symbol of an adapted pair. We use some elements of the terminology used in [22], transposed for the framework of adapted pairs (bivector-endomorphism), and we rewrite the results.

We consider an adapted pair $(w, J)$ where the minimal polynomial of $J$ has the form:

$$
\mu_{J}=\prod_{I=1}^{p}\left(X-\lambda_{I}\right)^{r_{I}} \prod_{I^{\prime}=1}^{q}\left(X^{2}-2 \alpha_{I^{\prime}} X+\alpha_{I^{\prime}}^{2}+\beta_{I^{\prime}}^{2}\right)^{r_{I^{\prime}}^{\prime}}
$$

with $p$ pairwise distinct real eigenvalues: $\lambda_{1}, \ldots, \lambda_{p}$ and $2 q$ conjugate complex eigenvalues: $\alpha_{1} \pm i \beta_{1}, \ldots, \alpha_{q} \pm i \beta_{q}$.

If we introduce, for $I \in\{1, \ldots, p\}$ (resp. $\left.I^{\prime} \in\{1, \ldots, q\}\right)$, the set $F_{I}=\operatorname{ker}\left(J-\lambda_{I} I d\right)^{r_{I}}$ (resp. $\left.F_{I^{\prime}}^{\prime}=\operatorname{ker}\left(J^{2}-2 \alpha_{I^{\prime}} J+\alpha_{I i}^{2}+\beta_{I^{\prime}}^{2}\right)^{r_{I^{\prime}}^{\prime}}\right)$, we have the following decomposition

$$
\operatorname{supp} w=\bigoplus_{I=1}^{p} F_{I} \bigoplus_{I^{\prime}=1}^{q} F_{I^{\prime}}^{\prime}
$$

where $F_{I}=\bigoplus_{j=1}^{s_{I}} F_{I}^{j}$ with $\operatorname{dim} F_{I}^{j}=2 r_{I}^{j}$ where $r_{I}=r_{I}^{1} \geq r_{I}^{2} \geq \cdots \geq r_{I}^{s_{I}}$ and $F_{I^{\prime}}^{\prime}=$ $\bigoplus_{j^{\prime}=1}^{s_{I}^{\prime}} F_{I^{\prime}}^{\prime j^{\prime}}$ with $\operatorname{dim} F_{I^{\prime}}^{j^{\prime}}=4 r_{I^{\prime}}^{\prime j}$ where $r_{I^{\prime}}^{\prime}=r_{I^{\prime}}^{\prime 1} \geq r_{I^{\prime}}^{\prime 2} \geq \cdots \geq r_{I^{\prime}}^{\prime s_{I^{\prime}}^{\prime}}$

The multiplicity of the eigenvalue $\lambda_{I}$ (resp. $\left.\alpha_{I^{\prime}}+i \beta_{I^{\prime}}\right)$ is $\left(r_{I}^{1}, r_{I}^{2}, \ldots, r_{I}^{s_{I}}\right.$ ) (resp. $\left.\left(r_{I^{\prime}}^{\prime 1}, r_{I^{\prime}}^{\prime 2}, \ldots, r_{I^{\prime}}^{\prime s_{I^{\prime}}^{\prime}}\right)\right)$.

Definition 3.7. The symbol $\sigma$ of the adapted pair $(w, J)$ is the element

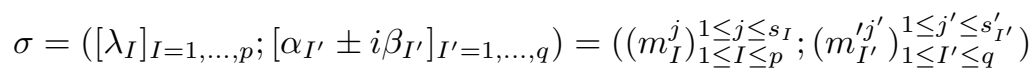

where $m_{I}^{j}=r_{I}^{j}$ and $m_{I^{\prime}}^{j^{\prime}}=2 r_{I^{\prime}}^{j^{\prime}}$.

3.1.5. Symbol stratification in $\mathcal{A}_{2 k}$. Transposing the results of [22] we get:

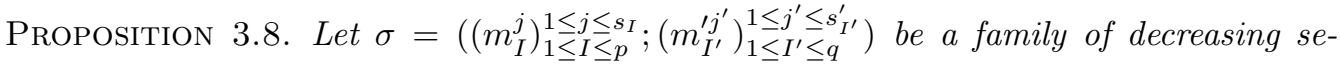
quences. If the subset $\Sigma^{\sigma}=\left\{(w, J) \in \mathcal{A}_{2 k}: \operatorname{symbol}(w, J)=\sigma\right\}$ is nonempty, it is a $G l(E)$-invariant submanifold of $\mathcal{A}_{2 k}$ of codimension

$$
N^{\sigma}-(2 k+2 q+p)
$$

where

$$
N^{\sigma}=3 \sum_{I=1}^{p} \sum_{j=1}^{s_{I}} j m_{I}^{j}+3 \sum_{I^{\prime}=p+1}^{q} \sum_{j^{\prime}=1}^{s_{I^{\prime}}} j^{\prime} m_{I^{\prime}}^{j^{\prime}}, \quad k=2 \sum_{I=1}^{p} \sum_{j=1}^{s_{I}} m_{I}^{j}+2 \sum_{I^{\prime}=p+1}^{q} \sum_{j^{\prime}=1}^{s_{I^{\prime}}} m_{I^{\prime}}^{j^{\prime}} .
$$

The sets $\Sigma_{c}^{\sigma}=\Sigma_{c} \cap \Sigma^{\sigma}$ constitute a $G l(E)$-invariant stratification of $\mathcal{A}_{2 k}$ which gives rise to sub-stratifications $\left\{\Sigma_{c}\right\}$ and $\left\{\Sigma^{\sigma}\right\}$ called refined stratification.

REMARK 3.9. The only open strata of the refined stratification of $\mathcal{A}_{2 k}$ are of the form $\Sigma_{0}^{\sigma}$ where we have for the symbol $\sigma: s_{I}=1$ for $I=1, \ldots, p$ and $s_{I^{\prime}}^{\prime}=1$ for $I^{\prime}=1, \ldots, q$.

3.2. Stratifications in the bundle $\mathcal{A}(M)$. Let $M$ be a manifold of dimension $n$.

Let $\mathcal{A}_{2 k}(M) \rightarrow M$ be the bundle of fibre $\mathcal{A}_{2 k}$ on which $G L(E)$ acts. Then $\mathcal{A}_{2 k}$ endowed with the stratification $\left\{\Sigma_{c}\right\}$, where each stratum $\Sigma_{c}$ is $G L(E)$-invariant, generates a stratification $\left\{\Sigma_{c}(M)\right\}$ on $\mathcal{A}_{2 k}(M)$. 
The space $\mathcal{A}_{2 k}(M)$ of smooth sections of this bundle, endowed with the smooth topology of Whitney, is a topological vector space and a Baire space. We shall denote by $\mathcal{A}(M)$ the union of the spaces of sections of $\mathcal{A}_{2 k}(M)$ for $0 \leq 2 k \leq n$.

In the same way, we get stratifications $\left\{\overline{\left.\Sigma^{\sigma}(M)\right\}}\right.$ (via the symbol) and $\left\{\Sigma_{c}^{\sigma}(M)\right\}$ on $\mathcal{A}_{2 k}(M) \simeq J^{0}\left(\underline{\mathcal{A}_{2 k}(M)}\right)$.

\section{Generic generalized PN manifolds}

4.1. Genericity. Let $\widetilde{\mathcal{A}}$ be the subset of the elements $(P, N)$ of $\underline{\mathcal{A}(M)}$ fulfilling the conditions:

$$
[P, P]=0, \quad T(N)=0 \quad \text { and } \quad[P, N]=0 .
$$

Let us recall that if $P$ is a Poisson tensor on $M$, the manifold $M$ is a union of immersed symplectic manifolds (cf. [29]) having the following properties on each symplectic leaf $F$ :

- $\operatorname{rank} P=2 k=\operatorname{dim} F$ and $\operatorname{supp} P=T F$,

- $N$ is an endomorphism $N_{F}$ of $T F$.

So $(P, N)$ gives rise on $F$ to a pair $\left(P_{F}, N_{F}\right)$ which is an element of $\widetilde{\mathcal{A}(F)}$.

Thom's transversality theorem (cf. [27]) indicates that the set $\tau \widetilde{\mathcal{A}(F)}$ of elements of $\widehat{\mathcal{A}(F)}$ whose 1 -jet is transverse to the stratification $\left\{\Sigma_{c}^{\sigma}(F)\right\}$, if nonempty, is a dense open set of $\widehat{\mathcal{A}(F)}$.

So we can associate by pull-back to any $(P, N)$ of $\widetilde{\mathcal{A}}$, giving rise to $\widetilde{\tau \mathcal{A}(F)}$, a stratification $\left\{\Sigma_{c}^{\sigma}(P, N)\right\}$ on $M$.

Definition 4.1. $(M, P, N)$ is called a generic generalized $P N$ manifold if $(P, N)$ generates an element of $\tau \widehat{\mathcal{A}(F)}$ on each symplectic leaf $F$.

In the next section, we shall construct examples of such generic pairs $(P, N)$.

REMARK 4.2. As $P$ is of constant rank $2 k$ on $F$, the genericity is only linked with the endomorphism $N_{F}$.

4.2. Geometry of a generic generalized PN manifold. We now describe the geometry of a generic generalized $\mathrm{PN}$ manifold.

TheOREM 4.3. Let $(M, P, N)$ be a generic generalized PN manifold. Consider

$$
\sigma=\left(\left(m_{1}^{1}, \ldots, m_{1}^{l_{1}}\right), \ldots,\left(m_{p}^{1}, \ldots, m_{p}^{l_{p}}\right),[\mu]\right) .
$$

Let $F$ be a $2 k$ dimensional symplectic leaf. If the stratum $\Sigma_{c}^{\sigma}(P, N)$ is nonempty, then $c=0$ or $c=2$ and it is a F-submanifold of codimension $c(c-1) / 2+N^{\sigma}-(2 k+2 q+p)$. Above this submanifold, $N$ has p pairwise distinct real eigenvalues and conjugate complex eigenvalues. One can find a decomposition in a Whitney sum $F_{H} \oplus F_{P, E}$ such that:

- on $F_{H}$, the operator only has complex eigenvalues,

- $F_{P, E}$ is a sum of bundles of the type $\operatorname{ker}\left(N-\lambda_{j} I d\right)^{r_{j}}$ and contains a Whitney sum of linear subspaces $\bigoplus_{j=1}^{s} \operatorname{ker}\left(N-\lambda_{\varphi(j)} I d\right)$ associated to the eigenvalues $\lambda_{\varphi(j)}$ where $\varphi(j) \in\{1, \ldots, p\}$.

REMARK 4.4. The nonempty submanifolds $\Sigma_{c}^{\sigma}$ of a leaf $F$ of dimension $2 k$ are: 
- open (resp. of codimension 1) for $\sigma=\underbrace{(1), \ldots,(1)}_{p \text { terms }} ; \underbrace{(2), \ldots,(2)}_{q \text { terms }}$ where $p \in E_{m}$ with $\left\{\begin{array}{ll}E_{m}=\{0,2, \ldots, m\} & \text { if } m \text { is even } \\ E_{m}=\{1,3, \ldots, m\} & \text { if } m \text { is odd }\end{array}\right.$ and $c=0($ resp. $c=2)$

- of codimension 1 (resp. 2) for $\sigma=\underbrace{(2),(1) \ldots,(1)}_{p-1 \text { terms }} ; \underbrace{(2), \ldots,(2)}_{q \text { terms }}$ where $p \in E_{m}^{\prime}$ with $\left\{\begin{array}{ll}E_{m}^{\prime}=\{2,4, \ldots, m\} & \text { if } m \text { is even } \\ E_{m}^{\prime}=\{3, \ldots, m\} & \text { if } m \text { is odd }\end{array}\right.$ and $c=0($ resp. $c=2)$

- of codimension greater than 2 in the other cases.

REMARK 4.5. In fact, a lot of strata are empty. This is due to the following reason: if one first considers the stratification by the rank of $P^{\prime}=N P$, and if one defines a notion of genericity for such Poisson tensors, one knows that, as in the case of forms ([17], [24]),

$$
\Sigma_{c}\left(P^{\prime}\right)=\left\{x \in M: \operatorname{rang} P_{x}^{\prime}=n-c\right\}
$$

is a submanifold of codimension $c(c-1) / 2$. Since the submanifold is a union of symplectic leaves of dimension $n-c$, one has $\operatorname{codim} \Sigma_{c}\left(P^{\prime}\right) \leq c$. So one generically gets nonempty strata for $c \geq c(c-1) / 2$, i.e. for $c \leq 3$. Now as $c=2 k-\operatorname{rang} P^{\prime}$, one necessarily has $c=0$ or $c=2$.

4.3. Examples of generic pairs. We now give two examples of generic pairs.

EXAmple 4.6. On $\mathbb{R}^{2 m}$ we consider the adapted pair $(P, N)$ defined by

$$
\begin{gathered}
P=\sum_{k=0}^{m-2} \partial_{2 k+1} \wedge \partial_{2 k+2}+x^{2 m-1} \partial_{2 m-1} \wedge \partial_{2 m} \\
N\left\{\begin{array}{c}
N \partial_{1}=\lambda_{1} \partial_{1} \\
N \partial_{2}=\lambda_{2} \partial_{2} \\
\vdots \\
N \partial_{2 m-3}=\lambda_{m-1} \partial_{2 m-3} \\
N \partial_{2 m-2}=\lambda_{m-1} \partial_{2 m-2} \\
N \partial_{2 m-1}=x^{2 m-1} \partial_{2 m-1} \\
N \partial_{2 m}=x^{2 m-1} \partial_{2 m}
\end{array}\right.
\end{gathered}
$$

where $0<\lambda_{1}<\cdots<\lambda_{m-1}$.

On the manifold $M=\left\{\left(x^{1}, \ldots, x^{2 m}\right):\left|x^{2 m-1}\right|<\lambda_{1}\right\}$ the symplectic leaves of the Poisson tensor are

- the open set $\Sigma_{0}: x^{2 m-1} \neq 0$,

- the submanifolds $L_{\alpha}$ of codimension 2 given by the equations $x^{2 m-1}=0$ and $x^{2 m}=\alpha$.

On $\Sigma_{0}$ the refined stratification is $\Sigma_{0}^{c}$ where $c=0,2$ and $\sigma=(1), \ldots,(1) ;(0)$ and one has codim $\Sigma_{2}^{\sigma}=1$. On $L_{\alpha}$ the symbol is still equal to $\sigma=(1), \ldots,(1) ;(0)$. So one gets a generic element.

ExAmple 4.7. One uses the example in $\mathbb{R}^{4}$ given in [2]. The open strata $\Sigma_{0}^{(1),(1) ;(0)}$ and $\Sigma_{0}^{(0) ;(2)}$ correspond respectively to $S^{E}$ and $S^{H}$ and where the stratum of codimension 1 , 
$\Sigma_{0}^{(2) ;(0)}$ corresponds to $S^{P}$. Here the Poisson tensor $P$ is constant

$$
P=\partial_{1} \wedge \partial_{2}+\partial_{3} \wedge \partial_{4}
$$

and the recursion operator $N$ is given, for $a \neq 0$, by its matrix in the canonical basis

$$
\left(\begin{array}{cccc}
a+x_{1} & 0 & 1 & 0 \\
0 & a+x_{1} & x_{4} & x_{3} \\
x_{3} & 0 & a & 0 \\
-x_{4} & 1 & 0 & a
\end{array}\right) .
$$

On the part which is defined by the inequality $x_{1}^{2}+4 x_{3} \geq 0$, the eigenfunctions $\lambda_{1}=$ $a+\frac{1}{2} x_{1}-\frac{1}{2} \sqrt{x_{1}^{2}+4 x_{3}}$ and $\lambda_{2}=a+\frac{1}{2} x_{1}+\frac{1}{2} \sqrt{x_{1}^{2}+4 x_{3}}$ of the operator $N$ are real. On the subset corresponding to $4 x_{3}+x_{1}^{2}>0$, one has $m_{N}=\left(X-\lambda_{1}\right)\left(X-\lambda_{2}\right)$ and the symbol is equal to $(1),(1) ;(0)$. The submanifold $\Sigma_{2}$ is of codimension 1 and its equation is $a x_{1}-x_{3}+a^{2}=0$. The subset of equation $4 x_{3}+x_{1}^{2}=0$ corresponds to a minimum polynomial $m_{N}=(X-\lambda)^{2}$ where $\lambda=a+\frac{1}{2} x_{1}$ and where $\sigma=(2) ;(0)$. The equations of the stratum $\Sigma_{2}^{(2) ;(0)}$ of codimension 2 are $x_{1}=-2 a, x_{3}=-a^{2}$.

\section{Existence of bi-abelian sub-algebra for a generic generalized PN manifold}

5.1. Properties of the momentum map relative to generic pairs. Let $(M, P, N)$ be a generalized PN manifold of dimension $2 m$ where $P$ is a Poisson tensor of maximal rank.

If we consider the functions $I_{k}=\frac{1}{k} \operatorname{trace} N^{k}(k=1, \ldots, m)$, the mapping

$$
x \mapsto\left(I_{1}(x), \ldots, I_{m}(x)\right)
$$

is called the momentum map (cf. [1]).

If $N$ only has pairwise distinct real eigenvalues, it is well known (cf. [15], [5] for example) that the functions $I_{1}, \ldots, I_{m}$ are pairwise in involution for both Poisson brackets $\{., .\}_{P}$ and $\{., .\}_{N P}$ and define a Lenard recursion chain, i.e.

$$
N^{*}\left(d I_{k}\right)=d I_{k+1}
$$

In particular $\left\{I_{1}, \ldots, I_{m}\right\}$ spans a bi-abelian sub-algebra.

We are going to prove that these properties are still true in a "generic" framework or in an analytic context.

Theorem 5.1. Let $(M, P)$ be a Poisson manifold of dimension $2 m$ where $P$ is a Poisson tensor of maximal rank. In the space $J_{1}\left(\mathcal{A}_{2 m}(M)\right)$ of the 1-jets of elements of $A_{2 m}(M)$ there exists a stratification such that if $(P, N)$ is transverse to it, one has the $\overline{\text { following }}$ properties (*):

- the functions $I_{k}, k=1, \ldots, m$ are independent on an open dense subset $\mathcal{O}$ of $M$ whose complement is a stratifiable set of codimension at least 2.

- In each Lie algebra $\left(\mathcal{C}(M),\{., .\}_{P}\right)$ and $\left(\mathcal{C}(M),\{., .\}_{N P}\right)$, the functions $I_{k}, k=$ $1, \ldots, m$, generate a bi-abelian Lie sub-algebra and constitute a Lenard recursion chain. 
If $(P, N)$ is analytic and if there exists a point of $M$ where the symbol is:

$$
\underbrace{(1), \ldots,(1)}_{p \text { terms }} ; \underbrace{(2), \ldots,(2)}_{q \text { terms }}
$$

with $p+2 q=m$ (for some $0 \leq p \leq m$ ), then there exists a dense open set $\mathcal{U}$ where the properties (*) hold.

REMARK 5.2. In the analytic case, if $N$ has only $m$ (pairwise distinct) double eigenvalues on an open dense subset $\mathcal{O}$ of $M$, and according to [12], the complement of $\mathcal{O}$ is an analytic set of codimension at least 2 . In this case the eigenfunctions $\lambda_{1}, \ldots, \lambda_{m}$ are defined on the whole manifold $M$ and are continuous, sub-analytic, nondifferentiable on $M \backslash \mathcal{O}$ but Lipschitz. In particular, such a generalized PN manifold will never be generic.

\subsection{Examples}

5.2.1. Example of a generic pair. We consider Example 4.7 again. Since the eigenvalues of the recursion operator are equal to

$$
\lambda_{1}=a+\frac{1}{2} x_{1}-\frac{1}{2} \sqrt{4 x_{3}+x_{1}^{2}} \text { and } \lambda_{2}=a+\frac{1}{2} x_{1}+\frac{1}{2} \sqrt{4 x_{3}+x_{1}^{2}}
$$

we can write

$$
\left\{\begin{array}{l}
I_{1}=\operatorname{trace} N=4 a+2 x_{1}, \\
I_{2}=\frac{1}{2} \operatorname{trace} N^{2}=2 x_{3}+a^{2}+\left(a+x_{1}\right)^{2} .
\end{array}\right.
$$

The equation of $\Sigma^{(2) ;(0)}$ is $4 x_{3}+x_{1}^{2}=0$ and the eigenvalues $\lambda_{1}$ and $\lambda_{2}$ are not differentiable on this submanifold.

On the contrary, the "first integrals" $I_{1}$ and $I_{2}$ are differentiable everywhere and independent because $d I_{1}=2 d x_{1}$ and $d I_{2}=4\left(a+x_{1}\right) d x_{1}+4 d x_{3}$

5.2.2. An example of nongenericity: the Hénon-Heiles system. We consider the example of the Hénon-Heiles system and we are interested in the second case of integrability where $\lambda=\frac{1}{2}$ and $B=4 A$. Using the coordinates: $x_{1}=q_{1}, x_{2}=q_{2}, x_{3}=p_{1}, x_{4}=p_{2}$, we consider the constant Poisson tensor

$$
P=\partial_{1} \wedge \partial_{3}+\partial_{2} \wedge \partial_{4}
$$

and the recursion operator $N$ given by its matrix

$$
\left(\begin{array}{cccc}
0 & x_{1} & 0 & 0 \\
x_{1} & 2 x_{2} & 0 & 0 \\
0 & -x_{3} & 0 & x_{1} \\
x_{3} & 0 & x_{1} & 2 x_{2}
\end{array}\right) .
$$

So the eigenvalues of $N$ are real and we have $\lambda_{1}=x_{2}+\sqrt{x_{1}^{2}+x_{2}^{2}}$ and $\lambda_{2}=x_{2}-\sqrt{x_{1}^{2}+x_{2}^{2}}$. They are sub-analytic, continuous and even Lipschitz. as quoted in Remark 5.2. On the complement of $S=\left\{x: x_{1}=x_{2}=0\right\}$ the symbol of $N$ is equal to (1), (1); (0); on $S$ we have $\sigma=(2) ;(0)$. Since $\operatorname{codim} S=2 \neq 1, N$ is not generic.

There does not exist any open subset where $\sigma=(0) ;(2)$, corresponding to a pair of conjugate complex eigenvalues.

Since the map $x=\left(x_{1}, x_{2}\right) \mapsto \sqrt{x_{1}^{2}+x_{2}^{2}}=\|x\|$ is not differentiable at 0 , neither $\lambda_{1}$ nor $\lambda_{2}$ are differentiable on $S$. 
According to the relations

$$
\left\{\begin{array}{l}
I_{1}=\operatorname{trace} N=4 x_{2}, \\
I_{2}=\frac{1}{2} \operatorname{trace} N^{2}=2 x_{1}^{2}+4 x_{2}^{2},
\end{array}\right.
$$

it is obvious that $I_{1}$ is differentiable on $S$ and $\left.d I_{1}\right|_{S}=0$. Similarly, $I_{2}$ is differentiable on $S$ and $\left.d I_{2}\right|_{S}=0$.

5.3. Construction of a suitable stratification. Let $M$ be a manifold of dimension $2 m$. We consider the case of the pairs $(P, N) \in \mathcal{A}_{2 m}(M)$, i.e. for $P$ of maximal rank $2 m$. We are to construct a stratification in the space of the 1-jets of elements of $\mathcal{A}_{2 m}(M)$.

Let $\pi_{1}$ be the canonical projection of the space $J_{1}\left(\mathcal{A}_{2 m}(M)\right)$ of 1-jets of elements $\mathcal{A}_{2 m}(M)$ on $\mathcal{A}_{2 m}(M)$. For $c=0,2, \widehat{\Sigma_{c}^{p}}(M)$ is the pull back $\pi_{1}^{-1}\left(\Sigma_{c}^{\tau}(M)\right)$ where

$$
\tau=\underbrace{(1), \ldots,(1)}_{p} ; \underbrace{(2), \ldots,(2)}_{q} \text { for } p \in E_{m}
$$

We then define the set $\widehat{\Sigma_{c}^{p, 1}}(M)$ of 1-jets of elements $(P, N)$ of $\underline{\mathcal{A}_{2 m}(M)}$ belonging to $\widehat{\Sigma_{c}^{p}}(M)$ such that

$$
d \lambda_{1} \wedge \cdots \wedge d \lambda_{p} \wedge d \alpha_{1} \wedge d \beta_{1} \wedge \cdots \wedge d \alpha_{q} \wedge d \beta_{q}=0
$$

where $\left\{\lambda_{j}\right\}_{j=1, \ldots, p}$ (resp. $\left\{\alpha_{k} \pm i \beta_{k}\right\}_{k=1, \ldots, q}$ ) are the real eigenvalues (resp. complex eigenvalues) of $N$ above $\Sigma_{c}^{\tau}(P, N)$.

Let $\widehat{\Sigma_{c}^{p, 0}}(M)$ be the complement of $\widehat{\Sigma_{c}^{p, 1}}(M)$ in $\widehat{\Sigma_{c}^{p}}(M)$. Moreover, let $\widehat{S^{p}}(M)$ be the pull back $\pi_{1}^{-1}\left(\Sigma_{0}^{\tau^{\prime}}(M)\right)$ where

$$
\tau^{\prime}=\underbrace{(2),(1), \ldots,(1)}_{p-1} ; \underbrace{(2), \ldots,(2)}_{q} \text { for } p \in E_{m}^{\prime}
$$

We first consider the set $\widehat{S^{p}, 2}(M)$ of 1-jets of elements of $\widehat{S^{p}}(M)$ which are not transverse to $\Sigma_{0}^{\tau^{\prime}}(M)$. We introduce the set $\widehat{S^{p, 1}}(M)$ of the elements of $\widehat{S^{p}}(M) \backslash \widehat{S^{p, 2}}(M)$ such that

$$
d \psi \wedge d \lambda_{2} \wedge \cdots \wedge d \lambda_{p} \wedge d \alpha_{1} \wedge d \beta_{1} \wedge \cdots \wedge d \alpha_{q} \wedge d \beta_{q}=0
$$

where $\left\{\lambda_{j}\right\}_{j=2, \ldots, p}$ (resp. $\left\{\alpha_{k} \pm i \beta_{k}\right\}_{k=1, \ldots, q}$ ) are the real eigenvalues (resp. conjugate complex eigenvalues) of $N$ above $\Sigma_{0}^{\tau^{\prime}}(P, N)$ whose local equation is $\psi=0$. We then consider the complement $\widehat{S^{p, 0}}(M)$ of $\widehat{S^{p, 1}}(M)$ in $\widehat{S^{p}}(M) \backslash \widehat{S^{p, 2}}(M)$.

Finally, we use $\widehat{\Sigma_{c}^{\sigma}}(M)=\pi_{1}^{-1}\left(\Sigma_{c}^{\sigma}(M)\right)$ for any $\sigma \neq \tau$ if $c=0,2$ and $\sigma \neq \tau^{\prime}$ if $c=0$.

Proposition 5.3. The following properties hold:

1. The sets $\widehat{\Sigma_{c}^{p, 1}}(M)$ for $c=0,2$ and $p \in E_{m}$ (resp. $\widehat{S^{p, 2}}(M)$ and $\widehat{S^{p, 1}}(M)$ for $p \in E_{m}^{\prime}$ ) are stratifiable and the codimension of the stratum of highest dimension is equal to at least 2 in $\widehat{\Sigma_{c}^{p}}(M)$ (resp. $\left.\widehat{S^{p}}(M)\right)$.

2. For $p \in E_{m}, \widehat{\Sigma_{0}^{p, 0}}(M)$ are open subsets of $J_{1}\left(\mathcal{A}_{2 m}(M)\right)$.

3. The sets $\widehat{\Sigma_{2}^{p, 0}}(M)$ for $p \in E_{m}$ and $\widehat{S^{p, 0}}(M)$ for $p \in E_{m}^{\prime}$ are submanifolds of codimension 1 in $J_{1}\left(\mathcal{A}_{2 m}(M)\right)$. 
4. If one chooses a stratification for each set $\widehat{\Sigma_{c}^{p, 1}}(M)$ for $c=0,2$ and $p \in E_{m}$, the union of these strata and the manifolds $\widehat{\Sigma_{0}^{p, 0}}(M)$ for $p \in E_{m}, \widehat{\Sigma_{2}^{p, 0}}(M)$ and $\widehat{S^{p, 0}}(M)$ for $p \in E_{m}^{\prime}, \widehat{\Sigma_{c}^{\sigma}}(M)=\pi_{1}^{-1}\left(\Sigma_{c}^{\sigma}(M)\right)$ for $\sigma \neq \tau$ if $c=0,2$ and $\sigma \neq \tau^{\prime}$ if $c=0$ is a stratification of $J_{1}\left(\mathcal{A}_{2 m}(M)\right)$.

5. If one considers a pair $(P, N)$ of $\mathcal{A}_{2 m}(M)$ transverse to the previous stratification, then the set $\mathcal{O}$ of the pull backs of the strata $\widehat{\Sigma_{0}^{p, 0}}(M)$ for $p \in E_{m}, \widehat{\Sigma_{2}^{p, 0}}(M)$ for $p \in E_{m}$ and $\widehat{S^{p, 0}}(M)$ for $p \in E_{m}^{\prime}$ is an open set of $M$ whose boundary $\partial \mathcal{O}$ is a stratified set of codimension at least 2 .

Sketch of the proof of Proposition 5.3. The different properties result from the construction of a stratification on a vector bundle obtained from a stratification on the fiber invariant under the action of a Lie group. More precisely, let $\widehat{\mathcal{A}_{2 m}}$ be the vector space of 1-jets at 0 of the pairs $(P, N)$ belonging to $\mathcal{A}_{2 m}\left(R^{2 m}\right)$ and let $\widehat{G}$ be the group of 2 -jets at 0 of diffeomorphism of $\mathbb{R}^{2 m}$ fixing 0 . The set $J_{1}\left(\mathcal{A}_{2 m}(M)\right)$ is a bundle on $M$ whose fiber over $x$ is isomorphic to $\mathcal{A}_{2 m}$ and has $\widehat{G}$ as structural group. The proof of the proposition is completed by the construction of $\widehat{G}$-invariant subsets of $\widehat{\mathcal{A}_{2 m}}$.

Sketch of the proof of Theorem 5.1. Let $(P, N)$ be transverse to the stratification constructed in Proposition 5.3 or an analytic pair fulfilling the assumptions of the theorem. Let us fix this pair and consider $\Sigma_{0}^{p}, \Sigma_{2}^{p}$ and $S^{p}$ the respective pull backs of the strata $\widehat{\Sigma_{0}^{p, 0}}$ for $p \in E_{m}, \widehat{\Sigma_{2}^{p, 0}}$, and $\widehat{S^{p, 0}}(M)$ for $p \in E_{m}^{\prime}$.

In the transversal case, according to Proposition 5.3, property 5, the union $\mathcal{V}$ of the sets $\left\{\Sigma_{c}^{p}\right\}_{c=0,2, p \in E_{m}}$ and $\left\{S^{p}\right\}_{p \in E_{m}^{\prime}}$ is an open dense set of $M$ whose boundary $\partial \mathcal{V}$ is a stratifiable set of codimension at least 2 . In the analytic case, these sets will be sub-analytic, and so stratifiable, and each $\Sigma_{0}^{p}$ is an open set.

It is easy to prove that the functions $I_{1}, \ldots, I_{m}$ are independent on $\Sigma_{0}^{p}$ where $N$ has $p$ double pairwise distinct real eigenvalues and $q$ different pairs of double conjugate complex eigenvalues.

The proof of the independence of $I_{1}, \ldots, I_{m}$ on $S^{p}$ and $\Sigma_{2}^{p}$ is left to the reader.

If $\Sigma_{0}$ is the (dense) open set, union of the open sets $\Sigma_{0}^{p}, p=0, \ldots, m$, we have established that the functions $I_{1}, \ldots, I_{m}$ are independent on the union $\mathcal{U}$ of the sets $\Sigma_{0}$, $\left\{S^{\prime p}\right\}_{p \in E_{m}^{\prime}}$ and $\left\{\Sigma_{c}\right\}_{p \in E_{m}}$.

By construction, $\mathcal{U}$ is the regular part of the pull-back of the set $\mathcal{O}$ defined in Proposition 5.3 , property 5 and which is equal to $\mathcal{V}$ in the transversal case. So the boundary of $\mathcal{U}$ is the union of stratifiable sets of codimension at least 2.

Now, as $L_{N X} N=N$ we can deduce (cf. [5]) that $N^{*} d I_{k}=d I_{k+1}$ and so $N^{*} d f_{l}=f_{l} d f_{l}$, $l=1, \ldots, m$. The proof of Theorem 5.1 is completed.

6. A characterization of the bi-abelian subalgebras. Let us consider a generalized PN manifold $(M, P, N)$ of dimension $n=2 m$ where $P$ is a Poisson tensor of maximal rank and $x$ a point of $M$ where the symbol of $(P, N)$ is equal to:

$$
\underbrace{(1), \ldots,(1)}_{p \text { terms }} ; \underbrace{(2), \ldots,(2)}_{q \text { terms }}
$$


with $p+2 q=m$. According to [28] and [23], one can find local coordinates on a neighborhood $U$ of $x$ such that

$$
P=\sum_{l=1}^{p} \frac{\partial}{\partial x_{l}} \wedge \frac{\partial}{\partial y_{l}}+\frac{1}{4} \sum_{l^{\prime}=1}^{q} \frac{\partial}{\partial u_{2 l^{\prime}-1}} \wedge \frac{\partial}{\partial u_{2 l^{\prime}}}-\frac{\partial}{\partial v_{2 l^{\prime}-1}} \wedge \frac{\partial}{\partial v_{2 l^{\prime}}}
$$

and where $N$ is characterized by:

$$
\begin{aligned}
N\left(\frac{\partial}{\partial x_{l}}\right) & =-\lambda_{l} \frac{\partial}{\partial x_{l}} \text { and } N\left(\frac{\partial}{\partial x_{l}}\right)=-\lambda_{l} \frac{\partial}{\partial x_{l}}, \quad l=1, \ldots, p \\
N\left(\frac{\partial}{\partial u_{2 l^{\prime}-1}}\right) & =\alpha_{l^{\prime}} \frac{\partial}{\partial u_{2 l^{\prime}-1}}+\beta_{l^{\prime}} \frac{\partial}{\partial v_{2 l^{\prime}-1}}, \quad N\left(\frac{\partial}{\partial v_{2 l^{\prime}-1}}\right)=-\beta_{l^{\prime}} \frac{\partial}{\partial u_{2 l^{\prime}-1}}+\alpha_{l^{\prime}} \frac{\partial}{\partial v_{2 l^{\prime}-1}} \\
N\left(\frac{\partial}{\partial u_{2 l^{\prime}}}\right) & =\alpha_{l^{\prime}} \frac{\partial}{\partial u_{2 l^{\prime}}}+\beta_{l^{\prime}} \frac{\partial}{\partial v_{2 l^{\prime}}}, \quad N\left(\frac{\partial}{\partial v_{2 l^{\prime}}}\right)=-\beta_{l^{\prime}} \frac{\partial}{\partial u_{2 l^{\prime}}}+\alpha_{l^{\prime}} \frac{\partial}{\partial v_{2 l^{\prime}}}, \quad l^{\prime}=1, \ldots, q
\end{aligned}
$$

where $\lambda_{1}, \ldots, \lambda_{p}, \alpha_{1} \pm i \beta_{1}, \ldots, \alpha_{q} \pm i \beta_{q}$ are the real and conjugate complex eigenvalues of $N$ on $U$ (it is possible to choose these coordinates such that $d y_{l}=d \lambda_{l}, l=1, \ldots, p$ and $\left.d u_{2 l^{\prime}}=d \alpha_{l^{\prime}} d v_{2 l^{\prime}}=d \beta_{l^{\prime}}, l^{\prime}=1, \ldots, q\right)$.

Such a coordinate system is called an adapted coordinate system.

Consider the hamiltonians $H_{1}, \ldots, H_{m}$, functionally independent on the open set $U$. $\left(H_{1}, \ldots, H_{m}\right)$ will be said to be separable in this set of coordinates if there exist $m$ relations, called separation relations, of the form $\left(\Phi_{1}, \ldots, \Phi_{p}, \Phi_{p+1}, \ldots, \Phi_{m}\right)$ :

$$
\begin{array}{ccc}
\Phi_{1}\left(x_{1}, y_{1}, H_{1}, \ldots, H_{m}\right) & = & 0, \\
\ldots \ldots \ldots . & \ldots & \ldots \\
\Phi_{p}\left(x_{p}, y_{p}, H_{1}, \ldots, H_{m}\right) & = & 0, \\
\Phi_{p+1}\left(u_{1}, u_{2}, H_{1}, \ldots, H_{m}\right) & = & 0, \\
\Phi_{p+2}\left(v_{1}, v_{2}, H_{1}, \ldots, H_{m}\right) & & \\
\ldots \ldots \ldots . & \ldots & \ldots \\
\Phi_{m-1}\left(u_{2 q-1}, u_{2 q}, H_{1}, \ldots, H_{m}\right) & = & 0, \\
\Phi_{m}\left(v_{2 q-1}, v_{2 q}, H_{1}, \ldots, H_{m}\right) & = & 0,
\end{array}
$$

with $\operatorname{det}\left[\partial \Phi_{l} / \partial H_{j}\right] \neq 0$ and the relations:

$$
\frac{\partial \Phi_{p+2 l^{\prime}-1}}{\partial u_{2 l^{\prime}-1}}=\frac{\partial \Phi_{p+2 l^{\prime}}}{\partial v_{2 l^{\prime}-1}}, \quad \frac{\partial \Phi_{p+2 l^{\prime}-1}}{\partial u_{2 l^{\prime}}}=\frac{\partial \Phi_{p+2 l^{\prime}}}{\partial v_{2 l^{\prime}}}, \quad l^{\prime}=1, \ldots, q .
$$

In this case, we get a generalization of a theorem given in [5] under the following assumptions:

There exists a point $x$ in $M$ where the symbol is

$$
\underbrace{(1), \ldots,(1)}_{p \text { terms }} ; \underbrace{(2), \ldots,(2)}_{q \text { terms }}
$$

for some $p \in\{0,1, \ldots, m\}$.

In this context, the set $\Sigma_{0}$ where the symbol is "maximal", i.e. (1), ., (1); (2), ., (2) for all $0 \leq p \leq m$, is an open dense set and, moreover, $P$ must have maximal rank on this set.

THEOREM 6.1. Under the previous assumptions, if $\left\{H_{1}, \ldots, H_{m}\right\}$ are functionally independent hamiltonian on an open set $V$ (which may be dense), the following properties are 
equivalent:

(1) on $\Sigma_{0} \cap V$ the m-uple $\left(H_{1}, \ldots, H_{m}\right)$ is separable in an adapted local coordinates system;

(2) the foliation defined by $\left(H_{1}, \ldots, H_{m}\right)$ on $V$ is lagrangian with respect to $P$ and invariant with respect to $N$;

(3) the functions $\left(H_{1}, \ldots, H_{m}\right)$ are in bi-involution on $V$.

In fact, these properties are local; so it is sufficient to prove the following result in an adapted local system on a neighborhood $U$ of $x \in V$ where the symbol of $(P, N)$ is:

$$
\underbrace{(1), \ldots,(1)}_{p \text { terms }} ; \underbrace{(2), \ldots,(2)}_{q \text { terms }}
$$

with $p+2 q=m$.

The proof of this theorem is an adaptation of the one given in [5] and is a consequence of the following observations.

ObSERvation 1. The property (1) holds iff there exists a square matrix $F=\left(F_{i j}\right)$ of order $m$ on $U$ with the same real and conjugate complex eigenvalues as $N$ fulfilling the property:

$$
N^{*}\left(d H_{i}\right)=\sum_{j=1}^{m} F_{i j} d H_{j}, \quad i=1, \ldots, m .
$$

OBSERVATion 2. Let $\left(H_{1}, \ldots, H_{m}\right)$ hamiltonians which define a Lagrangian foliation with respect to $P$ on $U$. Furthermore if this foliation is invariant with respect to $N$, there exists a square matrix $F=\left(F_{i j}\right)$ ) of order $m$ on $U$ with the same real and conjugate complex eigenvalues as $N$ with the property:

$$
N^{*}\left(d H_{i}\right)=\sum_{j=1}^{m} F_{i j} d H_{j}, \quad i=1, \ldots, m .
$$

ObSERvation 3. The properties (1) and (3) are equivalent.

\section{References}

[1] R. Abraham and J. E. Marsden, Foundations of Mechanics, 2nd ed., Perseus Book, 1978.

[2] P. Cabau and F. Pelletier, Localisation des courbes anormales et couples de tenseurs de Poisson en petites dimensions, Bulletin des Sciences Mathématiques 124 (2000), 459-515.

[3] R. Caboz, V. Ravoson and L. Gavrilov, Bi-hamiltoniaan structure of an integrable HénonHeiles system, J. Physics A: Math. Gen. 24 (1991), L523-L525.

[4] P. Damianou, Multiple Hamiltonian structures for Toda systems, J. Math. Phys. 35 (1994), 5511.

[5] G. Falqui and M. Pedroni, Separation of variables for bi-Hamiltonian systems, Math. Phys. Anal. Geom. 6 (2003), 139-179.

[6] G. Falqui, F. Magri, M. Pedroni and P. Zubelli, A bi-Hamiltonian theory for stationary KdV flows and their separability, Regul. Chaotic Dynam. 5 (2000), 33-52.

[7] H. Flaschka, Integrable systems and torus actions, in: Lectures on Integrable Systems, O. Babelon et al. (eds.), World Scientific, 1994, 43-101. 
[8] I. M. Gelfand and I. Zakharevich, On the local geometry of a bi-Hamiltonian structure, in: The Gel'fand Mathematical Seminars 1990-1992, L. Corwin et al. (eds.), Birkhäuser, Boston, 1993, 51-112.

[9] A. Ibort, F. Magri and G. Marmo, Bihamiltonian structures and Stäckel separability, J. Geom. Phys. 33 (2000), 210-228.

[10] B. Khesin and G. Misiolek, Euler equations on homogeneous spaces and Virasoro orbits, Adv. Math. 176 (2003), 116-144.

[11] Y. Kosmann-Schwarzbach and F. Magri, Poisson-Nijenhuis structures, Ann. Inst. Poincaré (Phys. Theor.) 53 (1990), 35-81.

[12] K. Kurdyka and L. Paunescu, Hyperbolic polynomials and multiparameter real analytic perturbation theory, Duke Math. J. 141 (2008), 123-149.

[13] F. Magri, A simple model of the integrable Hamiltonian equation, J. Math. Phys. 19 (1978), 1156-1162.

[14] F. Magri, G. Falqui and M. Pedroni, The method of Poisson pairs in the theory of non linear PDEs, in: Direct and Inverse Methods in Nonlinear Evolution Equations, Lecture Notes in Phys. 632, Springer, 2003, 85-136.

[15] F. Magri and C. Morosi, A geometrical characterization of integrable hamintonian systems through the theory of Poisson-Nijenhuis manifolds, Quaderno S 19, Università degli studi di Milano, 1984.

[16] F. Magri, C. Morosi and O. Ragnisco, Reduction techniques for infinite-dimensional Hamilton systems: some ideas and applications, Comm. Math. Phys. 99 (1985), 115-140.

[17] J. Martinet, Sur les singularités des formes différentielles, Annales de l'Institut Fourier (Grenoble) 20 (1970), no. 1, 95-178.

[18] C. Morosi and L. Pizzocchero, R-matrix theory, formal casimirs and the periodic Toda lattice, J. Math. Phys. 37 (1996), 4484-4513.

[19] J. M. Nunes da Costa and C.-M. Marle, Reduction of bihamiltonian manifolds and recursion operators, in: Differential Geometry and Applications (Brno, 1995), J. Janyška et al. (eds.), Masaryk University, Brno, 1996, 523-538.

[20] P. J. Olver, Applications of Lie Groups to Differential Equations, Graduate Texts in Mathematics 107, Springer, 2000.

[21] F. Pelletier, Singularités d'ordre supérieur de 1-formes, 2-formes et équations de Pfaff, Publications de l'I.H.E.S. 61 (1985), 129-169.

[22] F. Pelletier, Localisation des anormales en codimension 2 et variétés bipoissonniennes, Bulletin des Sciences Mathématiques 125 (2001), 197-234.

[23] F. Petalidou, Etude locale de structures bihamiltoniennes, Thèse, Université Pierre et Marie Curie, Paris VI, 1998.

[24] S. Pneumatikos, Structures symplectiques singulières génériques, Ann. Institut Fourier 34 (1984), 201-218.

[25] V. Ravoson, R. Caboz and L. Gavrilov, Structure Jacobi-hamiltonienne, Laboratoire de Physique Appliquée, Université de Pau et des Pays de l'Adour, Pau, 1992.

[26] A. Sergyeyev, A simple way of making a Hamiltonian system into a bi-Hamiltonian one, Acta Appl. Math. 83 (2004), 183-197.

[27] R. Thom, Un lemme sur les applications différentiables, Bul. Soc. Math. Mexico 1 (1956), $56-71$.

[28] F. J. Turiel, Classification locale simultanée d'un couple de deux formes symplectiques compatibles, Manuscripta Math. 82 (1994), 349-362.

[29] A. Weinstein, The local structure of Poisson manifolds, Journal of Differential Geometry 18 (1983), 523-557.

[30] K. Yano and S. Ishihara, Tangent and Cotangent Bundles: Differential Geometry, Marcel Dekker, New York, 1973. 
